Research Paper

\title{
Patterns of Failure and Survival Trends Of 720 Patients with Stage I Nasopharyngeal Carcinoma Diagnosed from 1990-2012: A Large-scale Retrospective Cohort Study
}

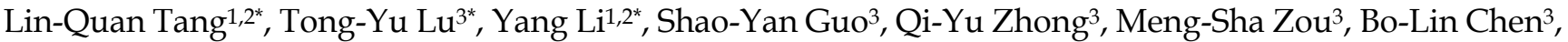 \\ Qing-Nan Tang1,2, Wen-Hui Chen" ${ }^{1}$, Shan-Shan Guo ${ }^{1,2}$, Li-Ting Liu1,2, Qiu-Yan Chen ${ }^{1,2}$, Ling Guo ${ }^{1,2}$, \\ Hao-Yuan Mo ${ }^{1,2}$, Rui Sun ${ }^{1,2}$, Dong-Hua Luo ${ }^{1,2}$, Chong Zhao ${ }^{1,2}$, Chao-Nan Qian ${ }^{1,2}$, Xiang Guo ${ }^{1,2}$, Mu-Sheng \\ Zeng ${ }^{1}$ and Hai-Qiang Mai ${ }^{1,2} \bowtie$ \\ 1. Sun Yat-sen University Cancer Centre, State Key Laboratory of Oncology in South China, Collaborative Innovation Centre for Cancer Medicine \\ 2. Department of Nasopharyngeal Carcinoma \\ 3. ZhongShan School of Medicine, Sun Yat-sen University \\ *these authors contributed equally to this work.
}

$\triangle$ Corresponding author: Mr. Hai-Qiang Mai, Department of Nasopharyngeal Carcinoma, Sun Yat-sen University Cancer Centre, 651 Dongfeng Road East, Guangzhou 510060, P. R. China; e-mail: maihq@sysucc.org.cn; Tel.: +86-20-87343380; Fax: +86-20-87343392

(C) Ivyspring International Publisher. This is an open access article distributed under the terms of the Creative Commons Attribution (CC BY-NC) license (https://creativecommons.org/licenses/by-nc/4.0/). See http://ivyspring.com/terms for full terms and conditions.

Received: 2017.05.14; Accepted: 2018.01.18; Published: 2018.03.23

\begin{abstract}
Objectives: To evaluate the patterns of failure and survival trends of patients with stage I nasopharyngeal carcinoma (NPC) treated with radiotherapy alone over the last 20 years.

Materials and Methods: A retrospective cohort study was conducted on 720 patients with stage I NPC who were treated with curative two-dimensional radiotherapy (2DRT), three-dimensional conformal radiotherapy (3DRT), or intensity-modulated radiotherapy (IMRT) between January 1990 and December 2012. The patients were categorized into four calendar periods (1990-1996, 1997-2002, 2003-2007, and 2008-2012) and four age subgroups (18-39, 40-49, 50-59, and >60). We computed overall survival (OS), progression free survival (PFS), locoregional relapse free survival (LRFS) and distant metastasis free survival (DMFS) as measures of patient survival.

Results: After a median follow-up period of 105 months (range 1-280 months), we observed the increasing trends in survival and disease control. The 3-, 5-, and 7-year OS rates increased from $97.0 \%$, $86.7 \%$, and $81.7 \%$ in the first calendar period (1990-1996) to $100 \%, 99.3 \%$, and $98.0 \%$ in the last calendar period (2008-2012), respectively $(P<0.001)$. Additionally, significant increasing trends could be seen in the PFS and LRFS during the four calendar periods. In the subgroup analysis, the OS, PFS and LRFS in patients diagnosed older than 40 years had greater improvement than the younger patients. However, the rate of distant metastasis was stable and relatively low, as the 5 -year distant metastasis rate ranged from $0.2 \%-2.5 \%$ among the four calendar periods.

Conclusion: The survival rates in patients with stage I NPC showed increasing trends from 1990 to 2012. The advances of radiotherapy provided excellent locoregional control and enhanced overall survival, and in particular, the IMRT decreased locoregional relapse.
\end{abstract}

Key words: Nasopharyngeal carcinoma; Radiotherapy; Prognosis

\section{Introduction}

Nasopharyngeal carcinoma (NPC) is an endemic disease in southern China and Southeast Asia, where the peak incidence is $0.5 \%$ [1]. NPC is characterized by the aggressive natural behaviours of early lymphatic spread and high preference of distant metastasis. Fortunately, radiotherapy (RT), the primary treatment, has been proven to be curative for NPC, especially in early stages. 
In 1965, Moss et al. established radiotherapy as the primary treatment for NPC [2]. Since then, conventional 2-dimensional radiotherapy (2DRT) was mainly used until 1990s. During the past two decades, with the accumulation of knowledge on radiobiology and target volume delineation, radiotherapy techniques have evolved from 2DRT to a 3-dimensional conformal technique (3DRT), and then to an intensity modulated technique (IMRT), which increased conformity of tumour coverage with better sparing of normal structures. Improvement of overall survival (OS) in patients with NPC in the last two decades was previously reported [3-7]: the 5-year OS rate was $50 \%$ in 1954-1992, and then increased to $77 \%$ in 1990-1999 and 85\% in 2000-2010. Nevertheless, the increase in survival outcome was attributed not only to the evolution of the radiotherapy technique but also to the development of imaging technology, which enhanced the accuracy of clinical staging and radiotherapy planning [8-11], the increasing use of more effective chemotherapy for patients with advanced stages [12], and better supportive care in recent years.

For the past 20 years, radiotherapy alone was the standard treatment for patients with stage I NPC. With the development of radiotherapy, we were interested in investigating the failure pattern and trend of survival and disease control during this time period. However, in previous studies with small numbers, a short time span, or short-term follow-up $[3,5,9,13-16]$, it was difficult to identify the significant difference in survival trends, or to discover convincing explanation for the benefits from the radiotherapeutic evolution. Thus, we conducted a large-scale retrospective analysis and a long-term follow-up, aiming to identify the failure pattern and trends of survival in patients with stage I NPC who received only radiotherapy treatment in 1990-2012.

\section{Material and methods}

\section{Patient Population}

A total of 720 patients with stage I NPC who received treatment between January 1990 and December 2012 at Sun Yat-sen University Cancer Centre were enrolled in this retrospective cohort study. This study was approved by the Clinical Research Ethics Committee of Sun Yat-sen University Cancer Centre, and written informed consent was provided by the participants. Details of the inclusion and exclusion criteria are available in Supplementary Materials. Pretreatment evaluations consisted of a medical history and a clinical examination of the head and neck region, blood chemistry tests, a computed tomography (CT)/magnetic resonance imaging (MRI) scan from the suprasellar cistern to the collarbone, fibreoptic nasopharyngoscopy, chest X-ray, abdominal sonography, and a whole-body bone scan or whole-body ${ }^{18}$ FDG-PET/CT (if available).

After the pretreatment evaluations, the following information was collected: sex, age, hereditary NPC, smoking status, BMI, and information on concurrent diseases, such as cardiovascular disease, diabetes mellitus (DM), or chronic HBV infection.

\section{Radiotherapy}

All patients with stage I NPC were treated with radiotherapy alone based on the principles of treatment for NPC patients at Sun Yat-sen University Cancer Centre. For the patients diagnosed in 2002 or earlier, the primary radiotherapy was 2DRT or 3DRT, and IMRT was also gradually performed in patients diagnosed in 2003 and later, in addition to 2DRT/3DRT. Previous publications have reported the three types of RT techniques in detail [17-19], which are presented in Supplementary Materials.

\section{Follow-up and Outcomes}

During the first three years after treatment, patients were followed up at least once every three months and then every six months thereafter until death. At each follow-up visit, we conducted a detailed history and a complete physical examination of the patient. When tumour relapse occurred, nasopharyngoscopy, MRI or CT of the head and neck, chest radiography, abdominal sonography, whole-body bone scan or PET/CT were performed.

\section{Statistics}

In the current study, we first explored OS, and then progression free survival (PFS), locoregional relapse free survival (LRFS) and distant metastasis free survival (DMFS). OS was calculated from the date of the first NPC diagnosis to the date of death from any cause or patient censoring at the date of the last follow-up. PFS was calculated from the date of the first NPC diagnosis to the date of relapse at any site, death from any cause or patient censoring at the date of the last follow-up. LRFS was calculated from the date of the first NPC diagnosis to the date of relapse at the head and neck region or patient censoring at the date of the last follow-up. DMFS was determined from the date of the first NPC diagnosis to the date of distant relapse or patient censoring at the date of the last follow-up. For patients diagnosed in 2002 or earlier, the primary radiotherapy was 2DRT or 3DRT, and IMRT was also gradually performed in patients diagnosed in 2003 and later, in addition to 2DRT/3DRT. So we divided the time period into four calendar periods: 1990-1996, 1997-2002, 2003-2007, and 2008-2012. The latter calendar period was made 
shorter than the previous one, to obtain a more even patient distribution and promote detection of survival effects arising from introduced changes in classification and therapy.

Categorical variables were classified based on clinical findings, and continuous variables were transformed into categorical variables based on routine cut-off points in clinical application [20,21].

SPSS (Statistical Package for the Social Sciences) 20.0 for Windows (SPSS, Armonk, New York, US) was used for statistical analyses. Survival curves were depicted using the Kaplan-Meier method and compared by the log-rank test. Chi-square test was conducted to evaluate the differences in categorical variables. All reported probability values were two-sided, and $P<0.05$ was considered to be statistically significant.

\section{Results}

A total of 720 consecutive patients with stage I NPC who received treatment between January 1990 and December 2012 at Sun Yat-sen University Cancer Center (550 males and 170 females; median age, 47 years; range, 18 to 97 years) were enrolled. Respectively, $98.2 \%, 94.1 \%, 90.3 \%$, and $84.3 \%$ of the patients had a complete follow-up of 3, 5, 7 and 10 years. The median follow-up period was 105 months (range 1-280 months). As shown in Table 1, there was no significant difference in the distributions of age, gender, EA-IgA1, comorbidities of DM, or CVD $(P>0.05)$ in the patients of the four calendar periods while there were significant differences for the imaging and radiotherapy techniques, body mass index (BMI), VCA-IgA1, smoking, comorbidities of chronic HBV infection and family history of NPC $(P<0.05)$.

Table 2 illustrated the details of the 3-, 5-, 7-, 10-, 15- and 20-year OS, PFS, LRFS and DMFS rates in the four calendar periods. Regarding the OS rate, the 3 -year OS rate remained relatively high $(>95 \%)$ during the four calendar periods, and the 5-year OS rate increased from $86.7 \%$ in the first period to $99.3 \%$ in the last calendar period. The 7- and 10-year OS rates also showed an increased trend (except that there was no data on the 10-year OS rate in 2008-2012). By 2007, the 10-year OS rate was up to $95.1 \%$. The increasing trends of the 3-, 5-, 7- and 10 -year OS rates were statistically significant in patients diagnosed before and after 2003. Similar results were found in the 3-, 5-, 7- and 10-year PFS rates and LRFS rates. For the DMFS rate, however, the 3-, 5-, 7- and 10- year DMFS rates remained high (ranging from $94.6 \%-100 \%$ ) during the four calendar periods, which indicated a low distant metastasis rate for stage I NPC patients.
In Table 3, the relapse details for patients of the four calendar periods were illustrated. In 1990-1996, $32(18.9 \%), 10(5.9 \%)$, and $7(4.1 \%)$ of 182 patients developed local relapse, regional relapse, and distant metastasis, respectively; in 1997-2002, 27 (14.4\%), 4 $(2.1 \%)$, and $9(4.8 \%)$ of 188 patients developed local relapse, regional relapse, and distant metastasis, respectively; in 2003-2007, 4 (2.3\%), $1(0.6 \%)$, and 3 $(1.7 \%)$ of 176 patients developed local relapse, regional relapse, and distant metastasis, respectively; in 2008-2012, $1(0.5 \%), 0(0 \%)$ and $3(1.6 \%)$ of 187 patients developed local relapse, regional relapse, and distant metastasis, respectively. From the statistics above, we found that the local control rate increased over time.

The OS, PFS, LRFS and DMFS curves on patients with stage I NPC diagnosed in the four calendar periods are shown in Figure 1A-D. We found that there was a remarkable improvement in OS, PFS, and LRFS of the patients diagnosed in 2003-2007 and 2008-2012, compared with those diagnosed in 1990-1996 and 1997-2002, which revealed that the differences of OS, PFS, and LRFS in patients among the four calendar periods were statistically significant $(\mathrm{P}<0.001)$. However, there was no significant difference in the patients' DMFS among the four calendar periods $(\mathrm{P}=0.620)$. Similar trends of the OS, PFS, LRFS and DMFS curves on male (Figure S1) and female (Figure S2) could be found in Supplementary Materials.

5-year OS, PFS, LRFS and DMFS rates of the four calendar periods subdivided by age-layers are shown in Figure 2A-D. The 5-year OS rate of patients in the age categories of 40-49, 50-59, and $\geq 60$ showed a rising trend. This increasing trend was, in particular, most obvious in patients over 60 years old (Figure 2A). The same trend could be found in the 5-year PFS and LRFS rates (Figure 2B\&C). For the patients in the age category of 18 to 39 years old, the 5-year OS, PFS, and LRFS rates were stable and quite high from 1990 to 2012. Figure 2D shows that the 5-year DMFS rate, regardless of age categories and calendar periods, remained stable and relatively high as well. Subdivided by genders, the 5-year OS, PFS, LRFS rates also showed increasing trends (Figure S3), which were presented in Supplementary Materials.

In subgroup analyses, the survival comparison between the 2DRT/3DRT arm and the IMRT arm, during the whole study period from 1990 to 2012, was shown in Supplementary Materials Figure S4. The remarkable improvement was found in OS, PFS, and LRFS of patients treated with IMRT, compared with those treated with 2DRT/3DRT, revealing that the differences were statistically significant $(\mathrm{P}<0.001)$, except for the DMFS. To address potential biases, we 
further conducted the analysis on the survival comparison between the 2DRT/3DRT arm and the IMRT arm, diagnosed during the years 2003 to 2012 (Figure 3), in which we found that only the LRFS was higher in the cohort of the IMRT arms (Figure 3C; $P<0.05)$ than that of the 2DRT/3DRT arms. No significant differences, however, were found in the OS, PFS, and DMFS rates between the IMRT and 2DRT/3DRT arms (Figure 3A, 3B, 3C; P>0.05). This result indicated that IMRT improved locoregional control thanks to radiation technique evolution.

\section{Discussion}

To our knowledge, this is the first study that has reported a retrospective large scale cohort study with a relatively long-term follow-up. A total of 720 patients with stage I NPC were enrolled in our study, and the follow-up lasted for over 20 years. It was undisputable that the prognosis of patients with stage I NPC had been significantly improved during the last two decades. Such improvement was prominently reflected in the increased trends of the OS, PFS, and LRFS rates (Table 2).

Table 1. Patient demographics and clinical characteristics

\begin{tabular}{|c|c|c|c|c|c|c|}
\hline & & 1990-1996 & 1997-2002 & 2003-2007 & 2008-2012 & \\
\hline Characteristics & No. (\%) & No. (\%) & No. (\%) & No. (\%) & No. (\%) & P value \\
\hline Age, $y$ & & & & & & 0.641 \\
\hline $18-39$ & $187(26.0)$ & $32(18.9)$ & $52(27.7)$ & $49(27.8)$ & $54(28.9)$ & \\
\hline $40-49$ & $233(32.4)$ & $59(34.9)$ & $60(31.9)$ & $54(30.7)$ & $60(32.1)$ & \\
\hline $50-59$ & $187(26.0)$ & $50(29.6)$ & $49(26.1)$ & $42(23.9)$ & $46(24.6)$ & \\
\hline$\geqslant 60$ & $113(15.7)$ & $28(16.6)$ & $27(14.4)$ & $31(17.6)$ & $27(14.4)$ & \\
\hline Sex & & & & & & 0.168 \\
\hline Female & $170(23.6)$ & $45(26.6)$ & $52(27.7)$ & $36(20.5)$ & $37(19.8)$ & \\
\hline Male & $550(76.4)$ & $124(73.4)$ & $136(72.3)$ & $140(79.5)$ & $150(80.2)$ & \\
\hline \multicolumn{7}{|l|}{ Clinical stage } \\
\hline T1N0M0, I & $720(100)$ & $169(23.4)$ & $188(26.1)$ & $176(24.4)$ & $187(26.1)$ & \\
\hline Imaging technique & & & & & & $<0.001$ \\
\hline CT & $385(53.5)$ & $169(100)$ & 180(95.7) & $35(19.9)$ & $1(0.5)$ & \\
\hline MRI & $308(42.8)$ & $0(0)$ & $8(4.3)$ & $135(76.7)$ & $165(88.2)$ & \\
\hline PET/CT+MRI & $27(3.8)$ & $0(0)$ & $0(0)$ & $6(3.4)$ & $21(11.2)$ & \\
\hline Radiotherapy technique & & & & & & $<0.001$ \\
\hline 2DRT/3DRT & $554(76.9)$ & $169(100)$ & $188(100)$ & $132(75.0)$ & $65(34.8)$ & \\
\hline TIMRT & $166(23.1)$ & $0(0)$ & $0(0)$ & $44(25.0)$ & $122(65.2)$ & \\
\hline Body mass index, $\mathrm{kg} / \mathrm{m} 2$ & & & & & & 0.010 \\
\hline$<18$ & $37(5.1)$ & $10(5.9)$ & $15(8.0)$ & $7(4.0)$ & $5(2.7)$ & \\
\hline $18-22.9$ & $356(49.4)$ & $98(58.0)$ & $98(52.1)$ & $71(40.3)$ & $89(47.6)$ & \\
\hline $22.9-27.4$ & $283(39.3)$ & $54(32.0)$ & $64(34.0)$ & $86(48.9)$ & $79(42.2)$ & \\
\hline$\geqslant 27.5$ & $44(6.1)$ & $7(4.1)$ & $11(5.9)$ & $12(6.8)$ & $14(7.5)$ & \\
\hline VCA-IgA1 & & & & & & $<0.001$ \\
\hline$\geqslant 1: 80$ & $528(73.5)$ & $141(83.4)$ & $158(84.0)$ & $115(65.3)$ & $115(61.5)$ & \\
\hline$<1: 80$ & $191(26.5)$ & $28(16.6)$ & $30(16.0)$ & $61(34.7)$ & $72(38.5)$ & \\
\hline EA-IgA1 & & & & & & 0.331 \\
\hline$\geqslant 1: 10$ & $397(55.1)$ & $95(56.2)$ & $113(60.1)$ & $93(52.8)$ & $96(51.3)$ & \\
\hline$<1: 10$ & $323(44.9)$ & $74(43.8)$ & $75(39.9)$ & $83(47.2)$ & $91(48.7)$ & \\
\hline Smoking & & & & & & 0.043 \\
\hline No & $448(62.2)$ & $96(56.8)$ & $110(58.5)$ & $111(63.1)$ & 131(70.1) & \\
\hline Yes & $272(37.8)$ & $73(43.2)$ & $78(41.5)$ & $65(36.9)$ & $56(29.9)$ & \\
\hline Diabetes mellitus & & & & & & 0.410 \\
\hline No & 706(98.1) & $166(98.2)$ & 186(98.9) & $170(96.6)$ & 184(98.4) & \\
\hline Yes & $14(1.9)$ & $3(1.8)$ & $2(1.1)$ & $6(3.4)$ & $3(1.6)$ & \\
\hline Chronic HBV infection & & & & & & 0.001 \\
\hline No & $689(95.7)$ & 168(99.4) & 184(97.9) & 161(91.5) & 176(94.1) & \\
\hline Yes & $31(4.3)$ & $1(0.6)$ & $4(2.1)$ & $15(8.5)$ & 11(5.9) & \\
\hline Cardiovascular disease & & & & & & 0.130 \\
\hline No & $684(95.0)$ & $163(96.4)$ & 183(97.3) & $164(93.2)$ & 174(93.0) & \\
\hline Yes & $36(5.0)$ & $6(3.6)$ & $5(2.7)$ & $12(6.8)$ & $13(7.0)$ & \\
\hline Family history of NPC & & & & & & 0.041 \\
\hline No & $634(88.1)$ & 157(92.9) & $165(87.8)$ & $146(83.0)$ & $166(88.8)$ & \\
\hline Yes & $86(11.9)$ & $12(7.1)$ & $23(12.2)$ & $30(17.0)$ & $21(11.2)$ & \\
\hline Total & $720(100)$ & $169(23.4)$ & $188(26.1)$ & $176(24.4)$ & $187(26.1)$ & \\
\hline
\end{tabular}

Abbreviations: $\mathrm{CT}=$ computed tomography; $\mathrm{MRI}=$ magnetic resonance imaging; $\mathrm{PET}=$ positron emission computer tomography; $2 \mathrm{DRT}=$ two-dimensional radiotherapy; $3 \mathrm{DRT}=$ three-dimensional conformal radiotherapy; IMRT = intensity-modulated radiotherapy; $\mathrm{EA}=$ early antigen; VCA = viral capsid antigen; IgA = immunoglobulin A; $\mathrm{HBV}=$ hepatitis $\mathrm{B}$ virus.

$P$ value was calculated with the Pearson $\chi^{2}$ test. 
Table 2. Suvival outcomes for the patients with stage I NPC in the four calendar periods

\begin{tabular}{|c|c|c|c|c|c|c|c|c|}
\hline & \multicolumn{2}{|c|}{ 1990-1996 } & \multicolumn{2}{|c|}{ 1997-2002 } & \multicolumn{2}{|c|}{ 2003-2007 } & \multicolumn{2}{|c|}{ 2008-2012 } \\
\hline & \multicolumn{2}{|c|}{$\%, 95 \%$ CI } & \multicolumn{2}{|c|}{$\%, 95 \%$ CI } & \multicolumn{2}{|c|}{$\%, 95 \%$ CI } & \multicolumn{2}{|c|}{$\%, 95 \%$ CI } \\
\hline \multicolumn{9}{|l|}{ OS } \\
\hline Rate at 3 year & 97.0 & $(94.5-99.5)$ & 95.7 & (92.8-98.6) & 100.0 & $(100.0-100.0)$ & 100.0 & $(100.0-100.0)$ \\
\hline Rate at 5 year & 86.7 & $(81.6-91.8)$ & 91.8 & $(87.9-95.7)$ & 98.8 & $(97.2-100.0)$ & 99.3 & $(97.9-100.0)$ \\
\hline Rate at 7 year & 81.7 & (75.8-87.6) & 87.2 & $(82.3-92.1)$ & 96.4 & (93.7-99.1) & 98.0 & $(95.3-100.0)$ \\
\hline Rate at 10 year & 75.1 & $(68.4-81.8)$ & 78.0 & $(71.7-84.3)$ & 95.1 & (91.4-98.8) & & \\
\hline Rate at 15 year & 61.3 & $(53.3-69.3)$ & 68.1 & $(60.7-75.5)$ & & & & \\
\hline Rate at 20 year & 48.8 & $(39.8-57.8)$ & & & & & & \\
\hline \multicolumn{9}{|l|}{ PFS } \\
\hline Rate at 3 year & 89.9 & $(85.4-94.4)$ & 89.8 & $(85.5-94.1)$ & 98.8 & $(97.2-100.0)$ & 99.5 & $(98.5-100.0)$ \\
\hline Rate at 5 year & 80.8 & $(74.9-86.7)$ & 85.9 & $(80.8-91.0)$ & 96.5 & $(93.8-99.2)$ & 98.0 & $(95.6-100.0)$ \\
\hline Rate at 7 year & 75.3 & $(68.8-81.8)$ & 80.8 & $(75.1-86.5)$ & 95.3 & $(92.2-98.4)$ & 96.7 & $(93.4-100.0)$ \\
\hline Rate at 10 year & 71.4 & $(64.5-78.3)$ & 75.1 & $(68.6-81.6)$ & 95.3 & $(92.2-98.4)$ & & \\
\hline Rate at 15 year & 57.5 & $(49.3-65.7)$ & 66.5 & $(59.1-73.9)$ & & & & \\
\hline Rate at 20 year & 48.1 & $(39.3-56.9)$ & & & & & & \\
\hline \multicolumn{9}{|l|}{ LRFS } \\
\hline Rate at 3 year & 91.6 & $(87.5-95.7)$ & 93.0 & $(89.3-96.7)$ & 98.8 & $(97.2-100.0)$ & 100.0 & $(100.0-100.0)$ \\
\hline Rate at 5 year & 85.6 & $(80.3-90.9)$ & 89.0 & $(84.5-93.5)$ & 97.1 & $(94.6-99.6)$ & 99.0 & $(97.2-100.0)$ \\
\hline Rate at 7 year & 79.8 & $(73.5-86.1)$ & 86.6 & $(81.5-91.7)$ & 96.5 & $(93.8-99.2)$ & 99.0 & $(97.2-100.0)$ \\
\hline Rate at 10 year & 77.7 & $(71.2-84.2)$ & 82.7 & $(77.0-88.4)$ & 96.5 & $(93.8-99.2)$ & & \\
\hline Rate at 15 year & 72.0 & $(64.6-79.4)$ & 80.6 & $(74.3-86.9)$ & & & & \\
\hline Rate at 20 year & 72.0 & $(64.6-79.4)$ & & & & & & \\
\hline \multicolumn{9}{|l|}{ DMFS } \\
\hline Rate at 3 year & 98.8 & $(97.2-100.4)$ & 97.8 & $(95.6-100.0)$ & 100.0 & $(100.0-100.0)$ & 99.5 & $(98.5-100.0)$ \\
\hline Rate at 5 year & 97.5 & $(95.0-100.0)$ & 97.8 & $(95.6-100.0)$ & 98.8 & $(97.2-100.4)$ & 98.3 & $(96.5-100.0)$ \\
\hline Rate at 7 year & 97.5 & (95.0-100.0) & 95.4 & (92.3-98.5) & 98.2 & $(96.2-100.2)$ & 98.3 & $(96.5-100.0)$ \\
\hline Rate at 10 year & 95.7 & (92.4-99.0) & 94.6 & (91.1-98.1) & 97.4 & $(94.9-99.9)$ & & \\
\hline Rate at 15 year & 95.7 & $(92.4-99.0)$ & 94.6 & (91.1-98.1) & & & & \\
\hline Rate at 20 year & 93.7 & $(88.6-98.8)$ & & & & & & \\
\hline
\end{tabular}

Table 3. Pattern of failure: the whole series and different groups

\begin{tabular}{lllll}
\hline & $\mathbf{1 9 9 0 - 1 9 9 6}$ & $\mathbf{1 9 9 7 - 2 0 0 2}$ & $\mathbf{2 0 0 3 - 2 0 0 7}$ & $\mathbf{2 0 0 8 - 2 0 1 2}$ \\
\hline & $\mathbf{n = 1 6 9 ( \% )}$ & $\mathbf{n = 1 8 8 ( \% )}$ & $\mathbf{n = 1 7 6}(\%)$ & $\mathbf{n = 1 8 7}(\mathbf{0})$ \\
\hline Local & $32(18.9 \%)$ & $27(14.4 \%)$ & $4(2.3 \%)$ & $1(0.5 \%)$ \\
regional & $10(5.9 \%)$ & $4(2.1 \%)$ & $1(0.6 \%)$ & $0(0 \%)$ \\
Distant & $7(4.1 \%)$ & $9(4.8 \%)$ & $3(1.7 \%)$ & $3(1.6 \%)$ \\
Local + regional & $0(0 \%)$ & $1(0.5 \%)$ & $0(0 \%)$ & $0(0 \%)$ \\
Distant + Local & $0(0 \%)$ & $0(0 \%)$ & $1(0.6 \%)$ & $0(0 \%)$ \\
Distant + regional & $0(0 \%)$ & $0(0 \%)$ & $0(0 \%)$ & $0(0 \%)$ \\
Distant + Local + regional & $0(0 \%)$ & $0(0 \%)$ & $0(0 \%)$ & $0(0 \%)$ \\
Overall & $49(29 \%)$ & $41(21.8 \%)$ & $9(5.1 \%)$ & $4(2.1 \%)$ \\
\hline
\end{tabular}

As shown in Figure 1C, the LRFS rate was much higher in 2003 or later, revealing the significant improvement of locoregional control in the latter two calendar periods. We considered that this improvement in LRFS was due to the gradual performance of IMRT in patients diagnosed in 2003 and later. Firstly, a remarkable improvement in LRFS of patients was shown in the analysis on survival comparison between the 2DRT/3DRT arm and the IMRT arm, during the whole study period from 1973 to 2008 (Figure S4). We further carried out a subgroup analysis on the survival comparison between the 2DRT/3DRT and the IMRT arm, restricted to patients diagnosed in 2003 and later (Figure 3), and found that IMRT also significantly improved the locoregional control. This is due to the higher radiation dose administered to reach the better tumour control with IMRT, compared with 2DRT/3DRT methods. The result was similar to other reports [3, 18, 22-27]. In other words, the IMRT changed the locoregional failure of the early stage NPC, which was the main failure pattern in the 2DRT/3DRT era (1990-2002) (Table 3).

The OS of patients with stage I NPC showed remarkable improvement, which was prominently attributed to the radiotherapy technique evolution, as shown in the current study and previous reports [3-5, 9, 13-16]. However, a subgroup analysis of the current study (Figure 3A), restricted to the patients diagnosed in 2003 and later, revealed that there were no significant differences in the OS between the 2DRT/3DRT and IMRT arms, which may be due to occult biases on the OS in the latter decade: salvage treatments including reirradiation [28], chemotherapy $[29,30]$, surgery after timely discovery of relapse with advances in imaging technology, or possibly, supportive care improvement. 
A

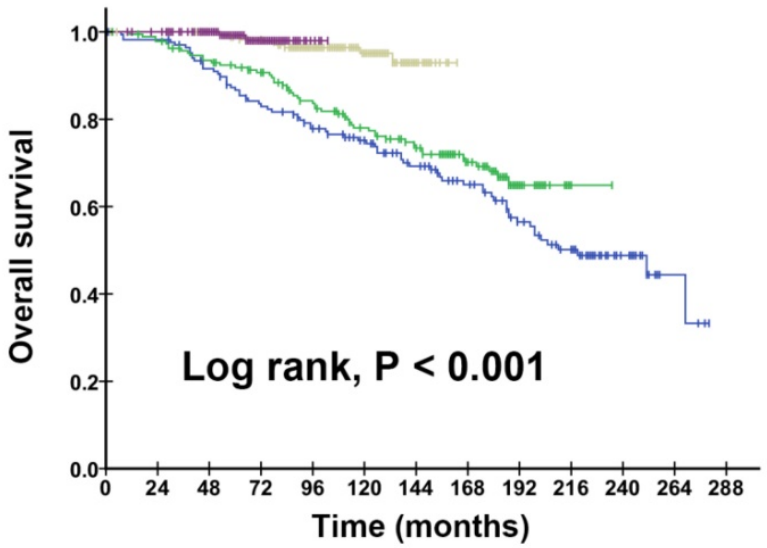

B

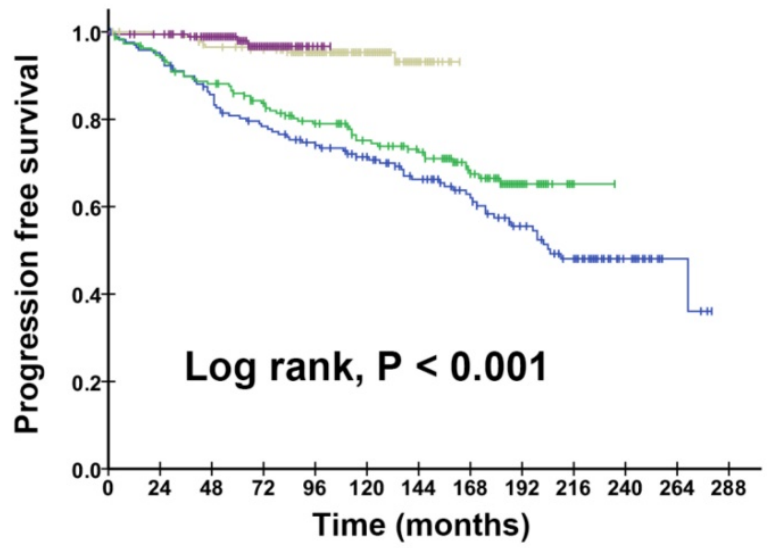

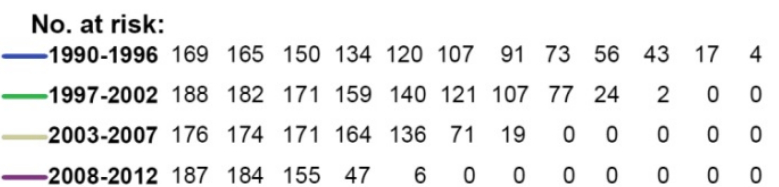

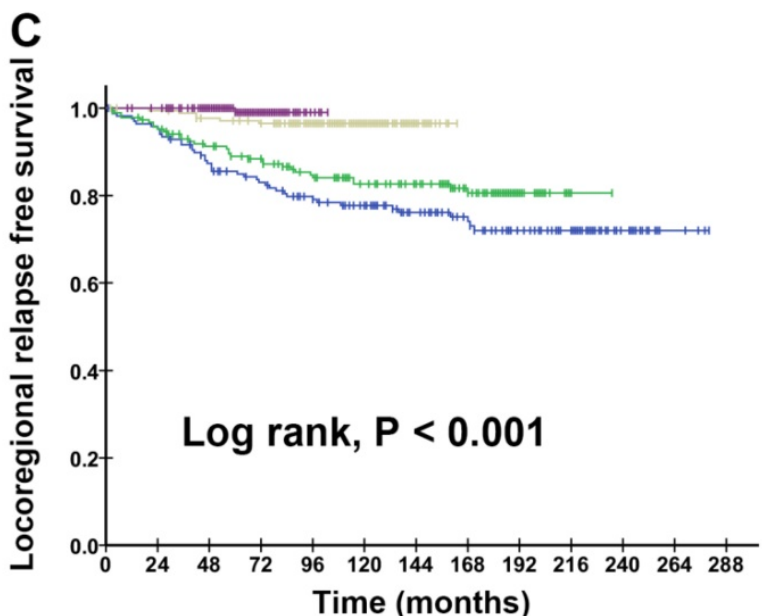

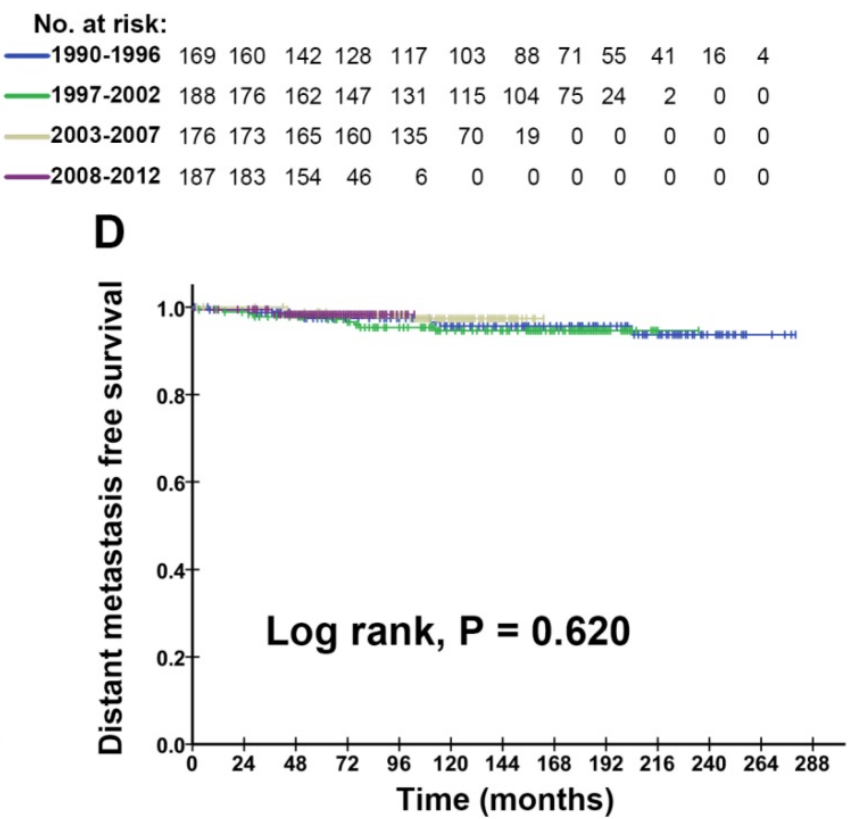

No. at risk:

$\begin{array}{llllllllllllll}-1990-1996 & 169 & 164 & 149 & 132 & 119 & 104 & 90 & 72 & 55 & 41 & 16 & 4\end{array}$

$\begin{array}{lllllllllllll}-1997-2002 & 188 & 181 & 171 & 57 & 138 & 120 & 107 & 77 & 24 & 2 & 0 & 0\end{array}$

-2003-2007 $176 \begin{array}{lllllllllll}174 & 169 & 161 & 135 & 70 & 19 & 0 & 0 & 0 & 0 & 0\end{array}$

-2008-2012 $187 \begin{array}{lllllllllll}183 & 154 & 47 & 6 & 0 & 0 & 0 & 0 & 0 & 0 & 0\end{array}$

Figure 1. Kaplan-Meier survival curves for the four calendar periods in the study population. Overall survival (A), progression free survival (B), locoregional relapse free survival $(C)$ and distant metastasis free survival $(D)$ compared in the entire cohort of stage I NPC patients. $P$ values were calculated using the unadjusted log-rank test.

One important issue is the stage migration phenomenon, which is inevitable with changing imaging techniques. From the statistics of our current study, CT was used as the main imaging technique in the first decade, accounting for $95.7 \%-100 \%$, but gradually, CT was replaced by MRI, which accounted for $80.1 \%-99.5 \%$ in the latter decade. The use of the PET/CT increased from $3.4 \%$ in $2003-2007$ to $11.2 \%$ in 2008-2012. As MRI has a higher resolution than CT, MRI worked better in the assessment of parapharyngeal spaces, marrow infiltration of the skull base, intracranial disease (advanced $\mathrm{T}$ stage), and deep cervical nodes (advanced $\mathrm{N}$ stage) [8].
PET/CT demonstrated greater accuracy in $\mathrm{N}$ and $\mathrm{M}$ staging compared to MRI [11], and the staging results, undoubtedly, affected the disease prognosis $[9,10]$. In the first decade when CT was used as the main imaging technique for staging, it is possible that patients with advanced T, N and M stage NPC, who, of course, had a poor prognosis, were diagnosed with stage I (T1N0M0) NPC. As the imaging techniques became more advanced, the staging of patients with NPC became more accurate, and thus, the evolution of imaging techniques could be considered as another factor that explained the uptrend of the OS, PFS, and LRFS in the last two decades. 
A

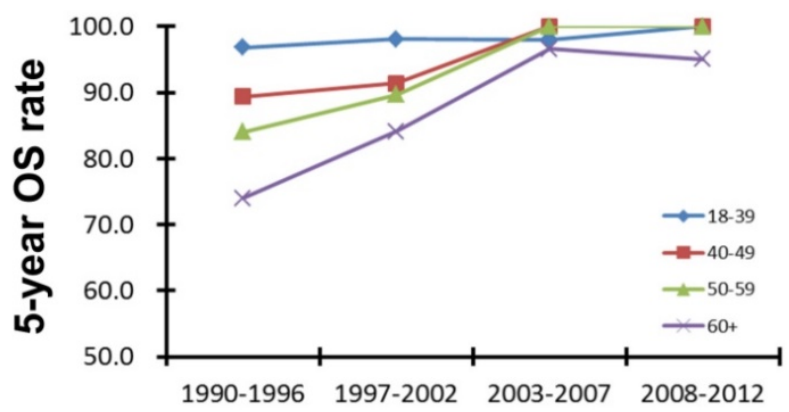

Calendar period

5-year overall survival rate $(95 \% \mathrm{Cl}), \%$

\begin{tabular}{|c|c|c|c|c|}
\hline Age category & 1990-1996 & $\underline{1997-2002}$ & 2003-2007 & 2008-2012 \\
\hline $18-39$ & $96.8(90.5-100.0)$ & $98.1(94.4-100.0)$ & $98.0(94.1-100.0)$ & $100.0(100.0-100.0)$ \\
\hline $40-49$ & $89.4(81.4-97.4)$ & $91.4(84.1-98.7)$ & $100.0(100.0-100.0)$ & $100.0(100.0-100.0)$ \\
\hline $50-59$ & $84.0(73.8-94.2)$ & $89.7(81.1-98.3)$ & $100.0(100.0-100.0)$ & $100.0(100.0-100.0)$ \\
\hline $60+$ & $74.0(57.3-90.7)$ & $84.1(69.8-98.4)$ & $96.6(89.9-100.0)$ & $95.0(85.4-100.0)$ \\
\hline
\end{tabular}

C

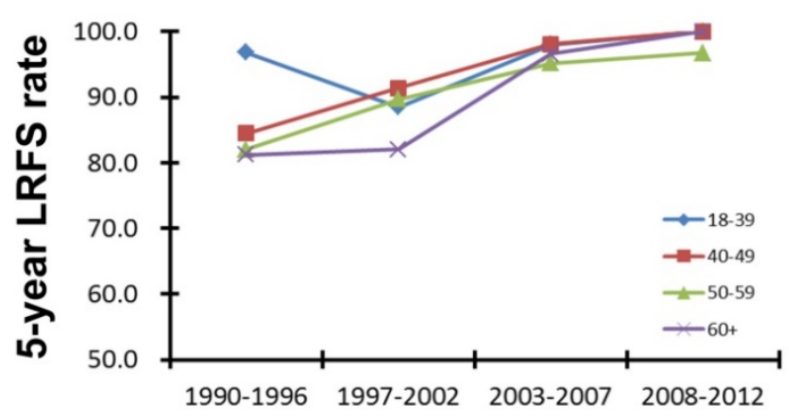

Calendar period

5-year locoregional relapse free survival rate $(95 \% \mathrm{Cl}), \%$

Age category 1990-1996 1997-2002 2003-2007 2008-2012

18-39 $\quad 96.8(90.5-100.0) \quad 88.5 \quad(79.9-97.1) \quad 98.0(94.1-100.0) \quad 100.0(100.0-100.0)$

40-49 $\quad 84.5(75.1-93.9) \quad 91.4(84.1-98.7) \quad 98.1(94.4-100.0) \quad 100.0(100.0-100.0)$

50-59 $\quad 82.0(71.4-92.6) \quad 89.7(81.3-98.1) \quad 95.2(88.7-100.0) \quad 96.7(90.2-100.0)$

$60+$

$\begin{array}{lllll}81.2(66.3-96.1) & 82.0 & (65.9-98.1) & 96.6(89.9-100.0) & 100.0(100.0-100.0)\end{array}$

Age

B

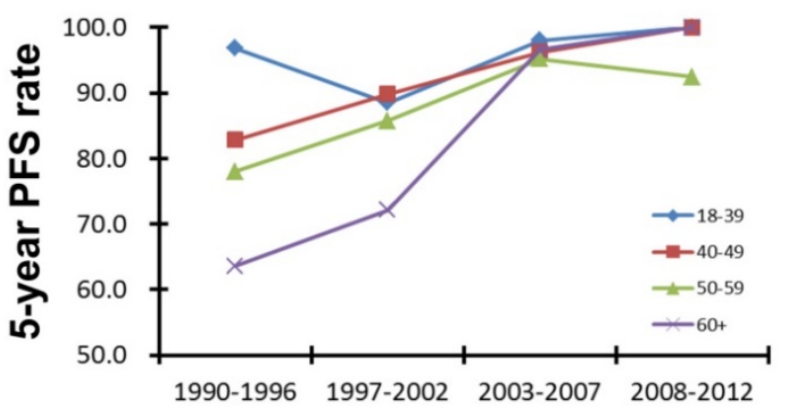

Calendar period

5-year progression free survival rate $(95 \% \mathrm{Cl}), \%$ Age category 1990-1996 1997-2002 2003-2007 2008-2012

$\begin{array}{lllll}18-39 & 96.8(90.5-100.0) & 88.5(79.9-97.1) & 98.0(94.1-100.0) & 100.0(100.0-100.0)\end{array}$

$\begin{array}{lllll}\mathbf{4 0 - 4 9} & 82.8(73.0-92.6) & 89.8(82.0-97.6) & 96.2(91.1-100.0) & 100.0(100.0-100.0)\end{array}$

$\begin{array}{lllll}\mathbf{5 0}-\mathbf{5 9} & 78.0(66.6-89.4) & 85.7(75.9-95.5) & 95.2(88.7-100.0) & 92.4(84.0-100.0)\end{array}$

$60+\quad 63.6(45.6-81.6) \quad 72.1(54.5-89.7) \quad 96.6(89.9-100.0) \quad 100.0(100.0-100.0)$

Figure 2. The 5-year survival rates for the four age subgroups in the four calendar periods. The 5-year overall survival rate (A), progression free survival rate (B), locoregional relapse free survival rate (C) and distant metastasis free survival rate (D) were shown in different age subgroups in 1990-1996, 1997-2002, 2003-2007, and 2008-2012.

Age is an important factor that influences NPC prognosis [20, 31]. From the analysis of the 5-year OS, PFS, LRFS, and DMFS rates subdivided by age groups among four calendar periods (Figure 2), the survival rates of patients diagnosed with NPC stage I age 18 to 39 years old were relatively high. In patients with NPC stage I over 40 years old, the OS, PFS, and LRFS rates showed increasing trends, from less than $90 \%$ in the first decade to over $95 \%$ in the latter decade. In Figure 2D, we observed that among the four calendar periods, the DMFS rates of patients in all of the age groups remained stable and high, at an average rate of over $90 \%$. In addition, from 2008 to 2012, the 5-year OS, PFS, LRFS, and DMFS rates of patients in all of the age categories were high. Therefore, we found that 1 ) for young patients (under the age of 40 years old), the calendar period was not an influencing factor for prognosis. In other words, young patients with stage I NPC had a higher survival rate whether they were treated with 2DRT or 3DRT; 2) the survival rates of patients with NPC stage I at an age of over 40 years old increased over time due to advanced techniques and better treatment. In particular, the survival rates of the elder patients (aged over 60) with stage I NPC 
A

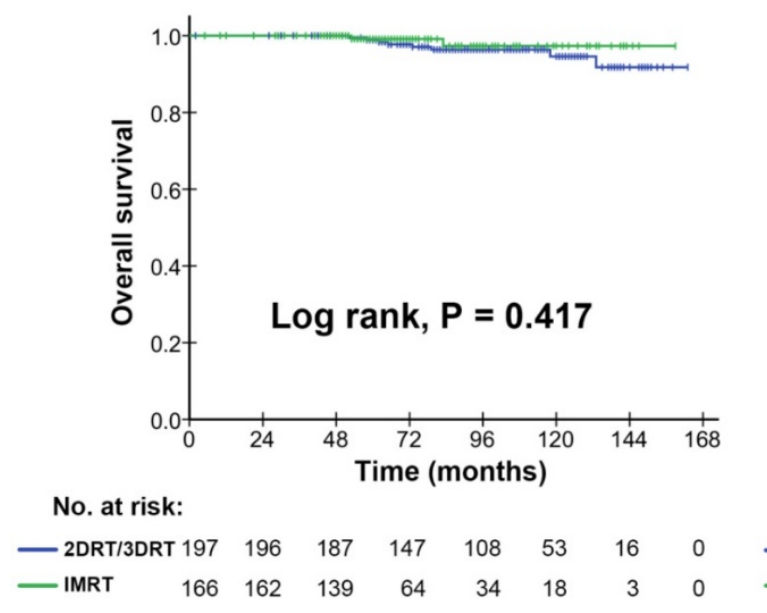

C

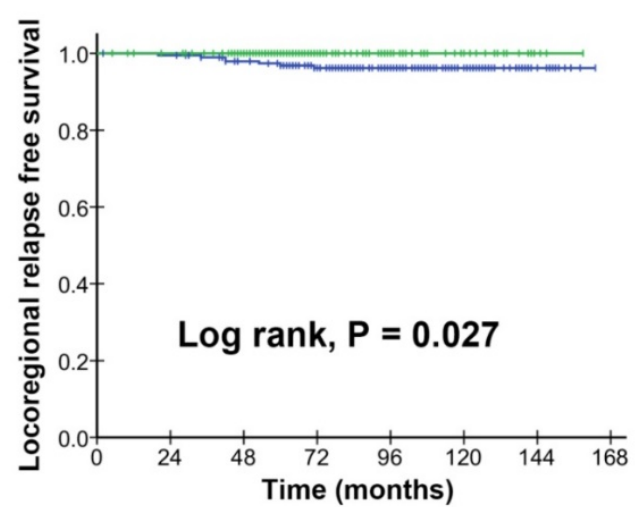

No. at risk:

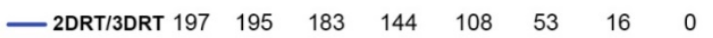

B

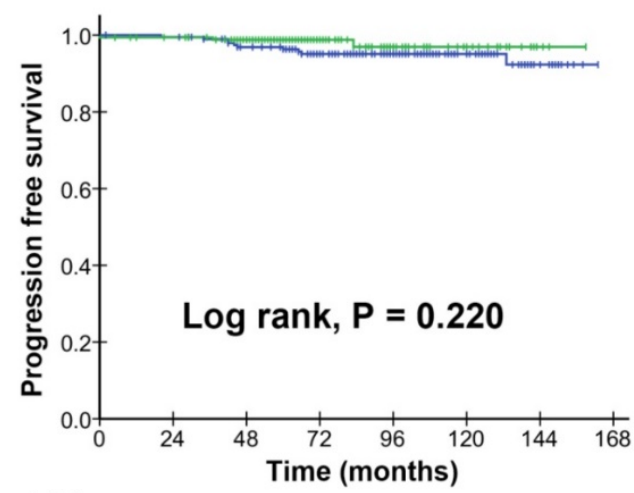

No. at risk:

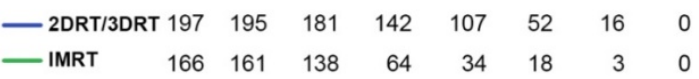

D

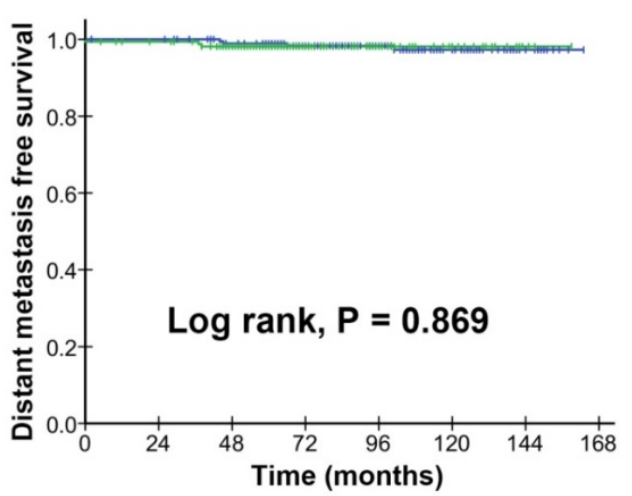

No. at risk:

$\begin{array}{llllllll}\text { - 2DRT/3DRT } 197 & 196 & 185 & 144 & 107 & 52 & 16 & 0\end{array}$

$\begin{array}{lllllllll}\text {-IMRT } & 166 & 161 & 138 & 64 & 34 & 18 & 3 & 0\end{array}$

Figure 3. Kaplan-Meier survival curves for the 2DRT/3DRT arm and IMRT arm in the patients diagnosed in 2003 and later. Overall survival (A), progression free survival (B), locoregional relapse free survival (C) and distant metastasis free survival (D) compared in the stage I NPC patients diagnosed in 2003 and later. P values were calculated using the unadjusted log-rank test.

could reach the same level as the young patients in 2008-2012; 3) the DMFS rates remained high for patients in all age groups among the four calendar periods, because distant metastasis rarely occurred in the patients diagnosed with NPC stage I.

The current study, however, still has its limitations. Firstly, the data were collected from a single institution. Besides, complete and accurate data about the late RT-toxicities were difficult to obtain as time passed by, so it was hard to know if rates of side effects were significantly less with IMRT. Thirdly, only patients with NPC stage I were included in the discussion, so this study cannot fully reflect the prognosis tendency and failure patterns of stage II-IV NPC among different treatment eras. Nevertheless, we have found promising results from the treatment progress of stage I NPC in the last 20 years, which encouraged us to conduct further studies on patients with stage II-IV NPC.

\section{Conclusions}

In our study, we observed that over the two decades from 1990 to 2012, the survival rates in patients with stage I NPC had significantly increased. The evolution of radiotherapy provided excellent locoregional control and enhanced overall survival, and in particular, the IMRT decreased locoregional relapse. In the modern era, the survival rates in patients with stage I NPC were high, and even the older patients could reach the same levels as the younger patients. We, therefore, appeal to a multicentre, long-term follow-up and large scale retrospective study to investigate NPC prognosis in the recent two decades.

\section{Supplementary Material}

Supplementary methods and figures. http://www.jcancer.org/v09p1308s1.pdf 


\section{Abbreviations}

NPC: nasopharyngeal carcinoma;

2DRT: two-dimensional radiotherapy; 3DRT:

threedimensional conformal radiotherapy; IMRT: intensity-modulated radiotherapy; OS: overall survival; PFS: progression free survival; LRFS: locoregional relapse free survival; DMFS: distant metastasis free survival; CT: computed tomography; MRI: magnetic resonance imaging; SPSS: Statistical Package for the Social Sciences.

\section{Acknowledgements}

We gratefully recognize the patients who participated in this study. This study was partly supported by the National Natural Science Foundation of China (NO.80425018, NO.81072226, NO.81201629, and NO. 81602371), the National Key Research and Development program of China (2016YFC0902000), the Special Support Plan of Guangdong Province (No.2014TX01R145), the SCI-Tech Project Foundation of Guangdong Province (No.2014A020212103, No.2011B080701034, No.2011B0 31800161), the Health \& Medical Collaborative Innovation Project of Guangzhou City (No. 201400000 001), the National Science \& Technology Pillar Program during the Twelfth Five-year Plan Period (No.2014BAI09B10), the PhD Start-up Fund of Natural Science Foundation of Guangdong Province, China (No. 2016A030310221), the cultivation foundation for the junior teachers in Sun Yat Sen University (No. 16ykpy28), the Sun Yat-sen University Clinical Research 5010 Program, the Sun Yat-sen University Cancer Center Clinical Research 308 Program, and the Fundamental Research Funds for the Central Universities.

\section{Competing Interests}

The authors have declared that no competing interest exists.

\section{References}

1. Wee JTS, Ha TC, Loong SLE, Qian CN. Is nasopharyngeal cancer really a "Cantonese cancer"? Chinese Journal of Cancer. 2010; 29: 517-26.

2. Moss WT. Therapeutic Radiology. 2nd ed. St Louis: C.V. Mosby; 1965.

3. Sun X, Su S, Chen C, Han F, Zhao C, Xiao W, et al. Long-term outcomes of intensity-modulated radiotherapy for 868 patients with nasopharyngeal carcinoma: An analysis of survival and treatment toxicities. Radiotherapy and Oncology. 2014; 110: 398-403.

4. Lee AW, Ng WT, Chan LL, Hung WM, Chan CC, Sze HC, et al. Evolution of treatment for nasopharyngeal cancer--success and setback in the intensity-modulated radiotherapy era. Radiother Oncol. 2014; 110: 377-84.

5. Yi JL, Gao L, Huang XD, Li SY, Luo JW, Cai WM, et al. Nasopharyngeal carcinoma treated by radical radiotherapy alone: Ten-year experience of a single institution. Int J Radiat Oncol Biol Phys. 2006; 65: 161-8.

6. Geara FB, Sanguineti G, Tucker SL, Garden AS, Ang KK, Morrison WH, et al. Carcinoma of the nasopharynx treated by radiotherapy alone: determinants of distant metastasis and survival. Radiother Oncol. 1997; 43: 53-61.

7. Lee AW, Poon YF, Foo W, Law SC, Cheung FK, Chan DK, et al. Retrospective analysis of 5037 patients with nasopharyngeal carcinoma treated during 1976-1985: overall survival and patterns of failure. Int J Radiat Oncol Biol Phys. 1992; 23: 261-70.
8. Lai V, Li X, Lee VHF, Lam KO, Fong DYT, Huang B, et al. Nasopharyngeal carcinoma: Comparison of diffusion and perfusion characteristics between different tumour stages using intravoxel incoherent motion MR imaging. European Radiology. 2014; 24: 176-83.

9. Lee AWM, Sze WM, Au JSK, Leung SF, Leung TW, Chua DTT, et al. Treatment results for nasopharyngeal carcinoma in the modern era: The Hong Kong experience. International Journal of Radiation Oncology Biology Physics. 2005; 61: 1107-16.

10. Yang Z, Shi Q, Zhang Y, Pan H, Yao Z, Hu S, et al. Pretreatment 18 F-FDG uptake heterogeneity can predict survival in patients with locally advanced nasopharyngeal carcinoma--a retrospective study. Radiation Oncology. 2015; 10: 4 .

11. Vellayappan BA, Soon YY, Earnest A, Zhang Q, Koh WY, Tham IWK, et al. Accuracy of 18F-flurodeoxyglucosepositron emission tomography/computed tomography in the staging of newly diagnosed nasopharyngeal carcinoma: A systematic review and meta-analysis. Radiology and Oncology. 2014; 48: 331-8.

12. Chen YP, Wang ZX, Chen L, Liu X, Tang LL, Mao YP, et al. A Bayesian network meta-analysis comparing concurrent chemoradiotherapy followed by adjuvant chemotherapy, concurrent chemoradiotherapy alone and radiotherapy alone in patients with locoregionally advanced nasopharyngeal carcinoma. Annals of Oncology. 2015; 26: 205-11.

13. Chua DT, Sham JS, Kwong DL, Au GK. Treatment outcome after radiotherapy alone for patients with Stage I-II nasopharyngeal carcinoma. Cancer. 2003; 98 : 74-80.

14. Ma J, Mai HQ, Hong MH, Cui NJ, Lu TX, Lu LX, et al. Is the 1997 AJCC staging system for nasopharyngeal carcinoma prognostically useful for Chinese patient populations? Int J Radiat Oncol Biol Phys. 2001; 50: 1181-9.

15. Xiao WW, Han F, Lu TX, Chen CY, Huang Y, Zhao C. Treatment outcomes after radiotherapy alone for patients with early-stage nasopharyngeal carcinoma. Int J Radiat Oncol Biol Phys. 2009; 74: 1070-6.

16. Kwong DL, Pow EH, Sham JS, McMillan AS, Leung LH, Leung WK, et al. Intensity-modulated radiotherapy for early-stage nasopharyngeal carcinoma: a prospective study on disease control and preservation of salivary function. Cancer; 2004. p. 1584-93.

17. Ma J, Mai HQ, Hong MH, Min HQ, Mao ZD, Cui NJ, et al. Results of a prospective randomized trial comparing neoadjuvant chemotherapy plus radiotherapy with radiotherapy alone in patients with locoregionally advanced nasopharyngeal carcinoma. J Clin Oncol. 2001; 19: 1350-7.

18. Lai SZ, Li WF, Chen L, Luo W, Chen YY, Liu LZ, et al. How does intensity-modulated radiotherapy versus conventional two-dimensional radiotherapy influence the treatment results in nasopharyngeal carcinoma patients? International Journal of Radiation Oncology Biology Physics. 2011; 80: 661-8.

19. Lee AW, Ng WT, Hung WM, Choi CW, Tung R, Ling YH, et al. Major late toxicities after conformal radiotherapy for nasopharyngeal carcinoma-patientand treatment-related risk factors. Int J Radiat Oncol Biol Phys. 2009; 73: 1121-8.

20. Tang LQ, Li CF, Li J, Chen WH, Chen QY, Yuan LX, et al. Establishment and Validation of Prognostic Nomograms for Endemic Nasopharyngeal Carcinoma. J Natl Cancer Inst. 2016; 108.

21. Huang P-Y, Wang C-T, Cao K-J, Guo X, Guo L, Mo H-Y, et al. Pretreatment body mass index as an independent prognostic factor in patients with locoregionally advanced nasopharyngeal carcinoma treated with chemoradiotherapy: findings from a randomised trial. Eur J Cancer. 2013; 49: 1923-31.

22. Peng G, Wang T, Yang KY, Zhang S, Zhang T, Li Q, et al. A prospective, randomized study comparing outcomes and toxicities of intensity-modulated radiotherapy vs. conventional two-dimensional radiotherapy for the treatment of nasopharyngeal carcinoma. Radiotherapy and Oncology. 2012; 104: 286-93.

23. Zhang MX, Li J, Shen GP, Zou X, Xu JJ, Jiang R, et al. Intensity-modulated radiotherapy prolongs the survival of patients with nasopharyngeal carcinoma compared with conventional two-dimensional radiotherapy: A 10 -year experience with a large cohort and long follow-up. European Journal of Cancer. 2015; 51: 2587-95.

24. Lin S, Pan J, Han L, Guo Q, Hu C, Zong J, et al. Update report of nasopharyngeal carcinoma treated with reduced-volume intensity-modulated radiation therapy and hypothesis of the optimal margin. Radiother Oncol. 2014; 110: 385-9.

25. Yi J, Huang X, Gao L, Luo J, Zhang S, Wang K, et al. Intensity-modulated radiotherapy with simultaneous integrated boost for locoregionally advanced nasopharyngeal carcinoma. Radiat Oncol. 2014; 9: 56.

26. Wu F, Wang $\mathrm{R}$, Lu H, Wei B, Feng G, Li G, et al. Concurrent chemoradiotherapy in locoregionally advanced nasopharyngeal carcinoma: treatment outcomes of a prospective, multicentric clinical study. Radiother Oncol. 2014; 112: 106-11.

27. Ng WT, Lee MCH, Chang ATY, Chan OSH, Chan LLK, Cheung FY, et al. The impact of dosimetric inadequacy on treatment outcome of nasopharyngeal carcinoma with IMRT. Oral oncology. 2014; 50: 506-12.

28. Hua YJ, Han F, Lu LX, Mai HQ, Guo X, Hong MH, et al. Long-term treatment outcome of recurrent nasopharyngeal carcinoma treated with salvage intensity modulated radiotherapy. Eur J Cancer. 2012; 48: 3422-8. 
29. Foo KF, Tan EH, Leong SS, Wee JTS, Tan T, Fong KW, et al. Gemcitabine in metastatic nasopharyngeal carcinoma of the undifferentiated type. Annals of Oncology. 2002; 13: 150-6.

30. Ngeow J, Lim WT, Leong SS, Ang MK, Toh CK, Gao F, et al. Docetaxel is effective in heavily pretreated patients with disseminated nasopharyngeal carcinoma. Annals of Oncology. 2011; 22: 718-22.

31. Chua MLK, Wee JTS, Hui EP, Chan ATC. Nasopharyngeal carcinoma. The Lancet. 2016; 387: 1012-24. 